Results During the study period 64 patients underwent ICED extraction, had suspicion of IE and had available imaging data; of whom median age was $67.5(17)$ years and 52(81.3\%) were male. IE was caused by Staphylococcus species in 44 (68.8\%), 51(79.7\%) episodes were definite according to the Duke criteria and 13 Duke possible. Device removal was usually performed percutaneously in $48(75 \%)$, with surgical extraction in 16. Reported complications included lead fracture (1) and superior vena cava dissection (1). Median episode duration (diagnosis to completion of antibiotics) was 40.5 (23.75) days, diagnosis to extraction was 17(23) days and extraction to completion of antibiotic therapy was 14(17.75) days. All included patients had an echocardiogram prior to extraction, 41(64.1\%) had evidence of vegetation and of these, 32 were confined to the ICED lead whilst nine had endocardial involvement. Following extraction 43(67.2\%) underwent echocardiography after a median of $8(7)$ days, demonstrating residual vegetation on native structures in 11 (figure 1). Compared to those without evidence of persistent vegetation, the duration of antibiotic therapy following extraction $(20(10)$ vs 7.5(15.3) days) and imaging (28(19) vs 14 (13.8) days) was longer. Patients without evidence of vegetations on post extraction echocardiogram had similar episode duration to those who did not have further imaging $(38.5(23)$ vs 36(29) days). Twenty (31.3\%) were pacing dependent and either received temporary pacing or immediate re-implantation,

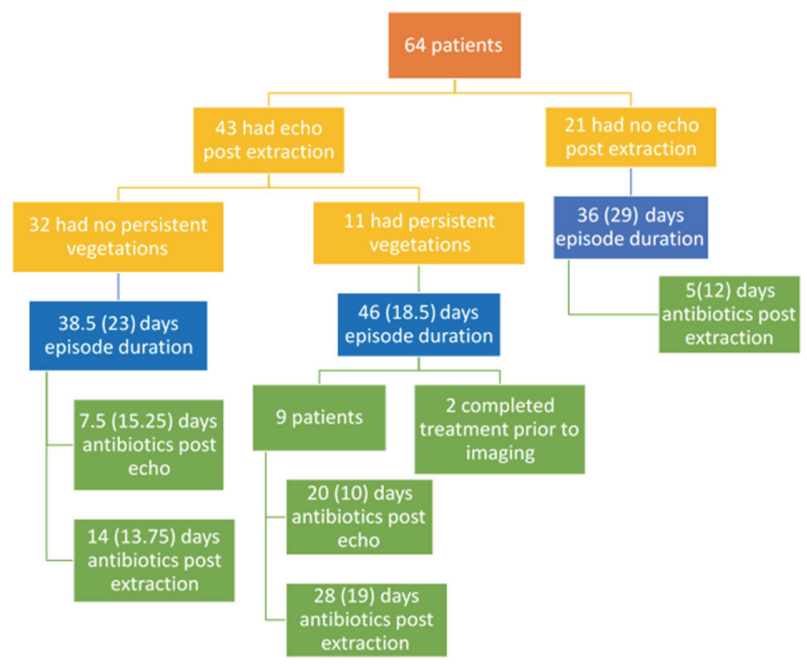

Abstract 171 Figure 1 Post ICED removal echocardiographic data and antibiotic therapy duration

Abstract 171 Table 1 Duration of antibiotic therapy in those with further ICED implant

\begin{tabular}{llll}
\hline & $\begin{array}{l}\text { Patients } \\
\text { (n) }\end{array}$ & $\begin{array}{l}\text { Antibiotics post } \\
\text { extraction (days) }\end{array}$ & $\begin{array}{l}\text { Duration of } \\
\text { episode (days) }\end{array}$ \\
\hline $\begin{array}{l}\text { Further ICED implant on } \\
\text { same admission }\end{array}$ & 37 & $14(17)$ & $42(24)$ \\
$\begin{array}{l}\text { Pacing dependent } \\
\text { Not pacing dependent }\end{array}$ & 20 & $11(17.5)$ & $43(23.25)$ \\
Discharged without ICED & 27 & $14(17)$ & $42(23)$ \\
\hline
\end{tabular}

and a further $37(57.8 \%)$ were re-implanted during the index presentation (table 1).

Conclusions In this series, in the absence of persistent vegetations on echocardiogram, after removal of an infected ICED, duration of antibiotic therapy was shorter. This suggests imaging can help guide course length, potentially reduce the length of hospital stays, antibiotic risks and selection for resistant bacteria.

Conflict of Interest No

\section{DETERMINING SYSTEMATIC BIAS IN THE ASSESSMENT OF LEFT VENTRICULAR FUNCTION BETWEEN TRANSTHORACIC ECHO AND CARDIAC MRI USING NEW GENERATION IMAGING SYSTEMS}

'Sally Chapman, 'Graham Fent, 'Laurence O'Toole, 'Oiver Watson, 'Jane Mackay, ${ }^{2}$ Pankaj Garg. 'Sheffield Teaching Hospitals NHS Trust, Sheffield, UK; ${ }^{2}$ Norfolk and Norwich University Hospitals

\subsection{6/heartjnl-2021-BCS.169}

Introduction Estimation of left ventricular systolic function (LVEF) commonly underpins clinical decision making in Cardiology. TTE has previously been demonstrated to underestimate LVEF compared with CMR. Sheffield Teaching Hospitals Trust (STH) recently upgraded to the latest generation of TTE scanners (GE E95) and CMR scanner (Siemens Magnetom Aera 1.5T). This service evaluation investigated correlation and bias between TTE and CMR LVEF reporting at our institution.

Methods Consecutive TTE studies at STH from April-October 2018 were cross-referenced, identifying patients who had also undergone CMR during this period. All patients - in and outpatients - with both TTE and CMR within 30 days were included. Patients with missing data, reversible aetiology or poor endocardial definition were excluded. The determination of LV function by echo was made using Simpson's biplane where endocardial definition was sufficient. If not, it was determined by visual assessment and a 5\% range quoted.

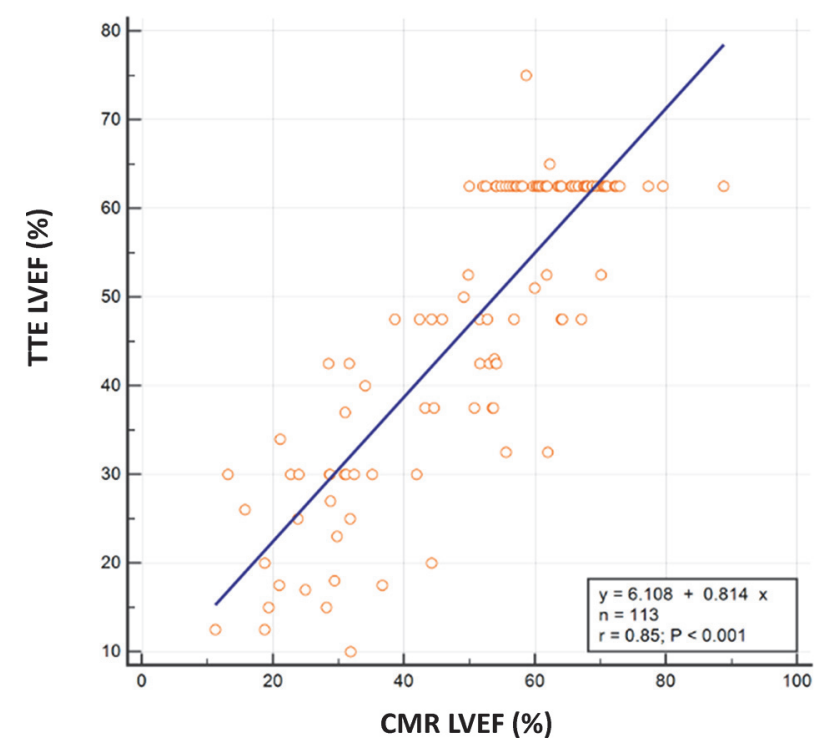

Abstract 172 Figure 1 
A second opinion was sought if LV function was assessed as $<40 \%$. LV function by cardiac MRI was determined by standard semi-automated endocardial contouring at end diastole and end systole using Siemens Syngovia software. The cut off for severe LV dysfunction was $<35 \%$ and $>55 \%$ for normal.

Results 12301 TTE and 398 CMR scans were performed in the study period. 120 patients met inclusion criteria with TTE and CMR within 30 days. Reasons for exclusion included missing data $(n=2)$, reversible cause for LV dysfunction $(n=1)$ and poor endocardial definition $(n=4)$ leaving a study population of 113 patients. LVEF by TTE and CMR (figure 1) were strongly correlated $(\mathrm{R}=0.85, \mathrm{p}<0.001)$. Bland Altman analysis demonstrated a signal of systematic bias with LVEF by CMR $4.4 \%$ higher than TTE (mean bias $4.4 \%, 95 \%$ CI -0.3 to $9.0, \mathrm{p}=0.06)$. Severe $\mathrm{LV}$ function on both TTE and CMR were in accordance in $91 \%$ of cases. Normal LV function by TTE and CMR was in accordance in $85 \%$ of cases.

Conclusion These results show good correlation of LVEF by TTE and CMR with the latest generation of scanners with a signal of systematic bias where CMR LVEF reads $4.4 \%$ higher than TTE. These results are used locally to guide clinical decision making and form part of on-going quality assurance processes. Further work to improve agreement between modalities is required.

Conflict of Interest None

\section{PREVALENCE OF ECHOCARDIOGRAPHIC ABNORMALITIES IN PATIENTS HOSPITALISED WITH COVID-19}

Dennis Chong, Issam Seyala, Muhammad Hamza, Sundes Shah, Khaled Makhlouf, WaiChung Wong, Gavin Bridgwood, Bethany Griffith, Diego Maseda. Mid Cheshire Hospitals NHS Foundation Trust, Crewe, UK

\subsection{6/heartjnl-2021-BCS.170}

Introduction COVID-19 is a multi-systemic disease and cardiac involvement has been widely recognised. Impaired cardiac function is linked to worse prognosis and has important implications on patient management. Evidence-based recommendations to guide selection of COVID-19 patients for transthoracic echocardiogram (TTE) are lacking, and there are concerns regarding unnecessary exposure of sonographers to infection risks.

Purpose 1. To determine the prevalence and nature of abnormal TTE findings in patients who underwent TTE within 6 months of admission to our centre with COVID-19 infection. 2. To determine if specific clinical characteristics were predictors of an abnormal TTE finding.

Methods A retrospective cohort study of all patients admitted to a single hospital with confirmed COVID-19 (through PCR) from $27 / 03 / 2020$ to $21 / 06 / 2020$, cross matched with the local TTE database.Data on patient demographics, TTE indication, biochemical markers, selected co-morbidities, ITU admission and mortality were collected. Patients with abnormal TTE were defined by the presence of left ventricular systolic dysfunction (LVSD), right ventricular systolic dysfunction (RVSD), regional wall motion abnormalities (RWMA) or pericardial effusion. Where available, patients with abnormal TTE were compared to previous TTE results to determine if findings

Abstract 173 Table 1
\begin{tabular}{|l|r|r|r|}
\hline Clinical characteristic & Abnormal TTE $(\mathbf{n}=31)$ & Normal TTE $(\mathbf{n}=39)$ & p value \\
\hline Age & $73 \pm 9.5$ & $70 \pm 14.0$ & 0.201 \\
\hline Sex & $58 \%$ & $45 \%$ & 0.344 \\
\hline CRP & $97 \pm 74$ & $117 \pm 123$ & 0.419 \\
\hline Lymphocyte count & $0.91 \pm 0.39$ & $0.84 \pm 0.47$ & 0.551 \\
\hline Raised HS-troponin* & $79 \%$ & $44 \%$ & 0.045 \\
\hline Hypertension & $46 \%$ & $36 \%$ & 0.466 \\
\hline Diabetes mellitus & $30 \%$ & $37 \%$ & 0.621 \\
\hline Ischaemic heart disease & $30 \%$ & $13 \%$ & 0.09 \\
\hline ITU admission & $16 \%$ & $26 \%$ & 0.383 \\
\hline Mortality & $34 \%$ & $18 \%$ & 0.18 \\
\hline *Data available for 37 patients
\end{tabular}

were new. If LVSD was previously present, any worsening in category of severity was considered significant. Unpaired t test and Fisher's exact test were used for between group statistical analysis.

Results We identified $71(12 \%)$ patients who underwent TTE from 597 patients admitted with a diagnosis of COVID-19. The mean age was $71(42-93)$ years and 37 (52\%) were male. 18 (25\%) patients died within the admission and 15 (21\%) required ITU care. The most frequent indications for TTE were suspected heart failure (42\%), endocarditis (17\%), MI (13\%), valvular dysfunction (7\%) and myopericarditis/effusion (7\%).A total of $33(46 \%)$ patients had an abnormal TTE. Of these, 30 (91\%) were new findings. LVSD was detected in 26 (37\%) cases: 5 (7\%) mild, 8 (11\%) moderate and $12(17 \%)$ severe. RVSD was detected in $12(17 \%)$, pericardial effusion in $9(13 \%)$ and RWMA in $10(14 \%)$. The only clinical characteristic that predicted an abnormal TTE was raised high sensitivity troponin (table 1). There was a trend towards an abnormal TTE in patients with known ischaemic heart disease $(30 \%$ Vs $13 \%, p=0.09)$. Increased mortality was observed in the abnormal TTE group (34\% Vs 18\%) although this did not reach statistical significance.

Conclusions There was a high prevalence of significant TTE abnormalities in patients admitted to our hospital with COVID-19 where TTE was clinically indicated. Our cohort had a predominance for LVSD rather than RVSD compared to other studies. Raised high sensitivity troponin level was a predictor of an abnormal TTE and may guide selection of COVID-19 patients for TTE.

Conflict of Interest None to declare

\section{CARDIAC MAGNETIC RESONANCE IMAGING IN PATIENTS WITH CHEST PAIN, TROPONIN INCREASE AND UNOBSTRUCTED CORONARY ARTERIES; A STUDY OF REAL-WORLD ADHERENCE TO GUIDELINES FROM A LARGE UK DISTRICT GENERAL HOSPITAL DURING THE COVID-19 PANDEMIC}

Oluwaseun Olubodun, Shereen Azeez, Karishma Dhera, Harriet Shearman, Andrew Elkington, Sacha Bull. Royal Berkshire Hospital, UK

\subsection{6/heartinl-2021-BCS.171}

Background The 2020 ESC Taskforce guidelines made a level I recommendation for the use of cardiac magnetic resonance (CMR) in patients with suspected myocardial infarction with non-obstructive coronary arteries (MINOCA). CMR is a powerful diagnostic tool that can discriminate between myocardial infarction, Takostubo and myocarditis; myocardial 